\title{
Elevated repulsive guidance molecule-a mRNA in peripheral blood mononuclear cells are associated with impaired leptomeningeal collaterals in patients with middle cerebral artery occlusions
}

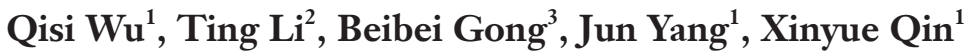 \\ ${ }^{1}$ Department of Neurology, The First Affiliated Hospital of Chongqing Medical University, Chongqing, China; ${ }^{2}$ Key Laboratory of Laboratory \\ Medical Diagnostics, Ministry of Education, Department of Laboratory Medicine, Chongqing Medical University, Chongqing, China; ${ }^{3}$ Department \\ of Radiology, The First Affiliated Hospital of Chongqing Medical University, Chongqing, China \\ Contributions: (I) Conception and design: Q Wu, X Qin; (II) Administrative support: None; (III) Provision of study materials or patients: B Gong, J \\ Yang; (IV) Collection and assembly of data: Q Wu, T Li; (V) Data analysis and interpretation: All authors; (VI) Manuscript writing: All authors; (VII) \\ Final approval of manuscript: All authors. \\ Correspondence to: Xinyue Qin. Department of Neurology, The First Affiliated Hospital of Chongqing Medical University, 1 Road Youyi, District \\ Yuzhong, Chongqing, China. Email: qinxinyuecq2008@sina.com.
}

Background: In the event of middle cerebral artery occlusion (MCAO), leptomeningeal collaterals (LMCs) play a crucial role in determining the survival of brain tissue distal to occlusion. Previous findings indicated that genes controlling arteriogenesis can impact the extent of LMCs. Therefore, probe for potential genetic parameters correlating of arteriogenesis may be clinically useful in predicting LMCs status in MCAO. During the screening process, we focused on repulsive guidance molecule a (RGMa), which has been reported to play a negative role in angiogenesis after stroke by decreasing the proliferation, migration, and tube formation of endothelial cells (ECs) in vivo and in vitro. Indeed, endothelial function plays a main role in arteriogenesis and is essential in determining the LMCs status. Therefore, in present study, we aimed to testify the hypothesis that RGMa might be associated with LMCs status in MCAO.

Methods: We prospectively enrolled patients with acute M1 MCA +/- intracranial internal carotid artery (ICA) occlusions $(n=96)$ and healthy controls $(n=33)$. Status of LMCs was evaluated according to computed tomographic angiography (CTA) on admission. Baseline RGMa mRNA expression was quantified by using quantitative real-time PCR.

Results: Patients with poor LMCs showed significantly higher RGMa mRNA levels than patients with good LMCs status $(\mathrm{P}=0.001)$ as well as healthy controls $(\mathrm{P}=0.002)$, respectively; whereas good LMCs group showed similar baseline RGMa levels than controls $(\mathrm{P}=1.000)$. RGMa mRNA level and baseline NIHSS score were independent predictors for impaired LMCs.

Conclusions: In MCAO patients, elevated PBMCs RGMa mRNA levels were associated with impaired LMCs status, indicating that measurement of RGMa mRNA expression in the early phase of stroke, together with other clinical approaches, was logically expected to be useful for predicting LMCs status. Moreover, a role for RGMa in leptomeningeal arteriogenesis following ischemic stroke can be hypothesized.

Keywords: Arteriogenesis; leptomeningeal collateral status (LMC status); middle cerebral artery occlusion (MCAO); peripheral mononuclear cells (PBMCs); repulsive guidance molecule a (RGMa)

Submitted Feb 14, 2020. Accepted for publication Jul 27, 2020.

doi: $10.21037 / \mathrm{apm}-20-422$

View this article at: http://dx.doi.org/10.21037/apm-20-422 


\section{Introduction}

In the setting of acute ischemic stroke (AIS) due to middle cerebral artery occlusion (MCAO), therapeutic decision making is greatly influenced by determination of prognosis for a potentially disabling neurologic deficit and how much brain is salvageable $(1,2)$. Indeed, penumbral 'life expectancy' in the event of MCA occlusion is greatly dependent on the status of collateral circulation, of which the leptomeningeal collaterals (LMCs) provides crucial nutritional support to the penumbra $(3,4)$. LMCs are preexisting anastomoses cross-connecting a small number of distal arterioles within the cerebral arterial tree. Previous studies have demonstrated that the LMCs status at baseline exhibits substantial variability among patients with $\mathrm{MCAO}$ and that patients with more collateral vessels have better outcomes, which made the LMCs status an important parameter in identifying patients with this devastating disease who may benefit from recanalization therapy $(5,6)$. However, imaging evaluation of human LMCs status does not directly visualize these small inter-arteriolar connections but instead relies on an indirect assessment of the extent and rate of backfilling of leptomeningeal arteries receiving blood flow through these collateral vessels $(2,3,6,7)$. Therefore, this is not a totally precise process based on information extracted only from radiologic assessment. It is necessary to search for better surrogates that enhance the accuracy of this determination.

Gene expression changes in peripheral mononuclear cells (PBMCs) have been observed in patients with ischemic stroke, suggesting these genes hold potential for diagnostic biomarker in these patients (8-10). Thus, probe for potential genetic parameters in PBMCs may be clinically useful in predicting the LMCs status in MCAO patients. Repulsive guidance molecule a (RGMa) is a glycosylphosphatidylinositol-anchored membrane protein expressed by a wide variety of cells, which has been involved in many physiopathological processes of the central nervous system (11-13). Recently, RGMa has been reported to play a negative role in angiogenesis after stroke by decreasing the proliferation, migration, and tube formation of endothelial cells (ECs) $(12,14)$. Indeed, endothelial function plays a main role in LMC formation (a process termed 'arteriogenesis'). Genetic variants impacting endothelial function could contribute to differences in LMCs status among individuals $(1,2,7$, 15-17). Therefore, we assumed that RGMa might be associated with LMCs status in patients with MCAO. In present study, we investigated the associations between RGMa mRNA levels in PBMCs and LMCs status in MCAO patients. We present the following article in accordance with the MDAR reporting checklist (available at http://dx.doi.org/10.21037/apm-20-422).

\section{Methods}

\section{Study population}

This study was a single-center prospective registered cohort trial (File No. ChiCTR1800018569) of patients aged 18 years or older presenting to the First Affiliated Hospital of Chongqing Medical University who were suspected of AIS in the first 24 hours of first found abnormal (FFA) during the study period (January 2018 to January 2019). All patients underwent a non-contrast computed tomography (NCCT) followed by computed tomographic angiography (CTA) of the head and neck at admission. And patients presenting with M1 segment of MCA +/- intracranial internal carotid artery (ICA) occlusion on baseline CTA were consecutively enrolled in the study. Patients with transient ischemic stroke, intracranial hemorrhage, severe systemic diseases including malignant tumors and autoimmune disease, acute or chronic infections, and suspicion of drug or alcohol abuse were excluded. During the same period, healthy subjects with no history of previous neurological disorders were recruited from the Physical Examination Center in the same hospital. They were matched with patients in terms of age and sex composition. Ethical approval for human subject research was obtained from the institutional review board of the participating hospital (File No. 2018-066-2) and written informed consent was provided by the participants or their proxy in accordance with Declaration of Helsinki (as revised in 2013).

Demographics, clinical history, stroke risk factors and laboratory results were collected on all participants. The subtypes of AIS were determined by Trial of Org 10172 in Acute Ischemic Stroke Treatment (TOAST) criteria (18). Stroke severity was quantified using National Institute of Health Stroke Scale (NIHSS) at admission. Pre-stroke modified Rankin scale (mRS) score was recorded for all patients and follow-up mRS was obtained at 3 months; a favorable functional outcome was defined as 90-day mRS $\leq 2$. According to the international stroke management guide the treatment protocol was applied. Additionally, treatment decision was made at the treating neurologist's 
discretion.

\section{Image acquisition and analysis}

CT scans were obtained with a multi-slice scanner (GE Discovery CT750HD, GE Healthcare, Wisconsin). Wholebrain NCCT was performed: $100 \mathrm{kV}, 120 \mathrm{mAs}$ and $5 \mathrm{~mm}$ slice thickness. Then CTA was performed by scanning from the cerebral vertex to the aortic arch, using the following parameters: slice thickness $5 \mathrm{~mm}$ and intravenous administration of $70-80 \mathrm{~mL}$ non-ionic contrast at $3-5 \mathrm{~mL} / \mathrm{s}$. CTA source images in three planes (axial, coronal, sagittal) were reconstructed using 20-mmthick slabs. All images were independently assessed by two experienced observers: a stroke neurologist and a neuroradiologist, who were blinded to clinical information. Final decisions were reached by a consensus. Reviewers assessed the status of LMCs on baseline CTA by using regional leptomeningeal collateral (rLMC) score system (20 points), which appeared to be a reliable and valid measure for defining LMCs status in AIS patients $(3,19)$. Leptomeningeal vascularity was dichotomized as $0-10$ (poor) and 11-20 (good) according to a previous study (6).

\section{Blood sampling and isolation of human PBMCs}

Peripheral venous blood samples (4-5 $\mathrm{mL})$ were obtained from the enrolled patients on arrival at the Emergency Department, whereas the blood samples from the controls were taken before their breakfast. By using Ficoll (TBD science, Tianjin, China) gradient centrifugation, PBMCs were isolated within 2 hours of blood draw and then were transferred into $1 \mathrm{~mL}$ TRIzol Reagent in $1.5 \mathrm{~mL}$ centrifuge tubes. PBMCs were stored at $-80^{\circ} \mathrm{C}$ until RNA extraction.

\section{Real-time reverse transcription polymerase chain reaction (RT-PCR)}

According to the manufacturer's protocol, total RNA was extracted from PBMCs by using TRIzol Reagent (Invitrogen, CA, USA) and then quantified with the NanoDrop ND-1000 (Thermo Scientifc, USA). Total RNA was reverse transcribed with a PrimeScript RT reagent Kit (Takara, Dalian, China). Then RT-PCR was carried out using SYBR Green PCR kit (Takara, Dalian, China) on an instrument of $\mathrm{T} 100^{\mathrm{TM}}$ Thermal Cycler (Bio-RAD, CA, USA). Briefly, cDNA was amplified with primers provided by Invitrogen (CA, USA) at $95^{\circ} \mathrm{C}$ for $3 \mathrm{~min}$, followed by
41 cycles of $30 \mathrm{~s}$ at $95{ }^{\circ} \mathrm{C}, 30 \mathrm{~s}$ at $50^{\circ} \mathrm{C}$ and $30 \mathrm{~s}$ at $72{ }^{\circ} \mathrm{C}$. mRNA primers were listed in as follows: RGMa: $5^{\prime}$-CGA GCT GGA TGG ATG GGT AT-3' and 5'-GGA AGC GGT CGG TGA AAG T-3'; GAPDH: 5'-ACG GAT TTG GTC GTA TTG GGC G-3' and 5'-CTC CTG GAA GAT GGT GAT GG-3'. By using the BioRad CFX Manager software, the data analysis was carried out based on the comparative cycle threshold $(\mathrm{Ct})$ method $\left(2^{-\Delta \Delta \mathrm{Ct}}\right)$ using GAPDH as the interval standard control. The results are based on three independent experiments.

\section{Statistical analysis}

Continuous data with normal distribution were presented as mean \pm standard deviation (SD) and were analyzed with Student's $t$-test or one-way ANOVA test. Continuous data with non-Gaussian distribution were described as medians (interquartile range, IQR) and were analyzed with MannWhitney U test or Kruskal-Wallis test. Categorical variables were recorded as numbers (\%) and were analyzed with Pearson Chi-Square test. A receiver-operating characteristic (ROC) curve analysis was used to determine the potential usefulness of RGMa for the prediction of poor LMCs status in AIS patients. We performed multivariate logistic regression analysis to evaluated independent factors for poor LMCs status and good outcome, respectively. Variables from univariate analyses at $\mathrm{P}<0.1$ were included in each multivariable model. The RGMa mRNA level was a priori forced into the model since it was the predictor of interest. A $\mathrm{P}$ value $<0.05$ (two-sided) was considered statistically significant. All data analyses were performed using SPSS version 23.0 (SPSS Inc., Chicago, Illinois, USA).

\section{Results}

\section{Baseline characteristics}

Between January 2018 and January 2019, 96 patients (42.7\% females, mean age $67.7 \pm 12.1$ years) were identified as having AIS due to M1 MCA +/- ICA occlusions were enrolled in the analysis. According to the CTA manifestations on admission, 45 (46.9\%) patients were graded as having good LMCs (rLMC score 11-20) and 51 (53.1\%) were graded as having poor LMCs (rLMC score 0-10) (Figure 1A,B). During the same period, 33 subjects were included as healthy controls (60.6\% females, mean age $62.8 \pm 10.6$ years). Among the three subgroups, there were no significant differences with respect to age, sex, obesity, smoking and 

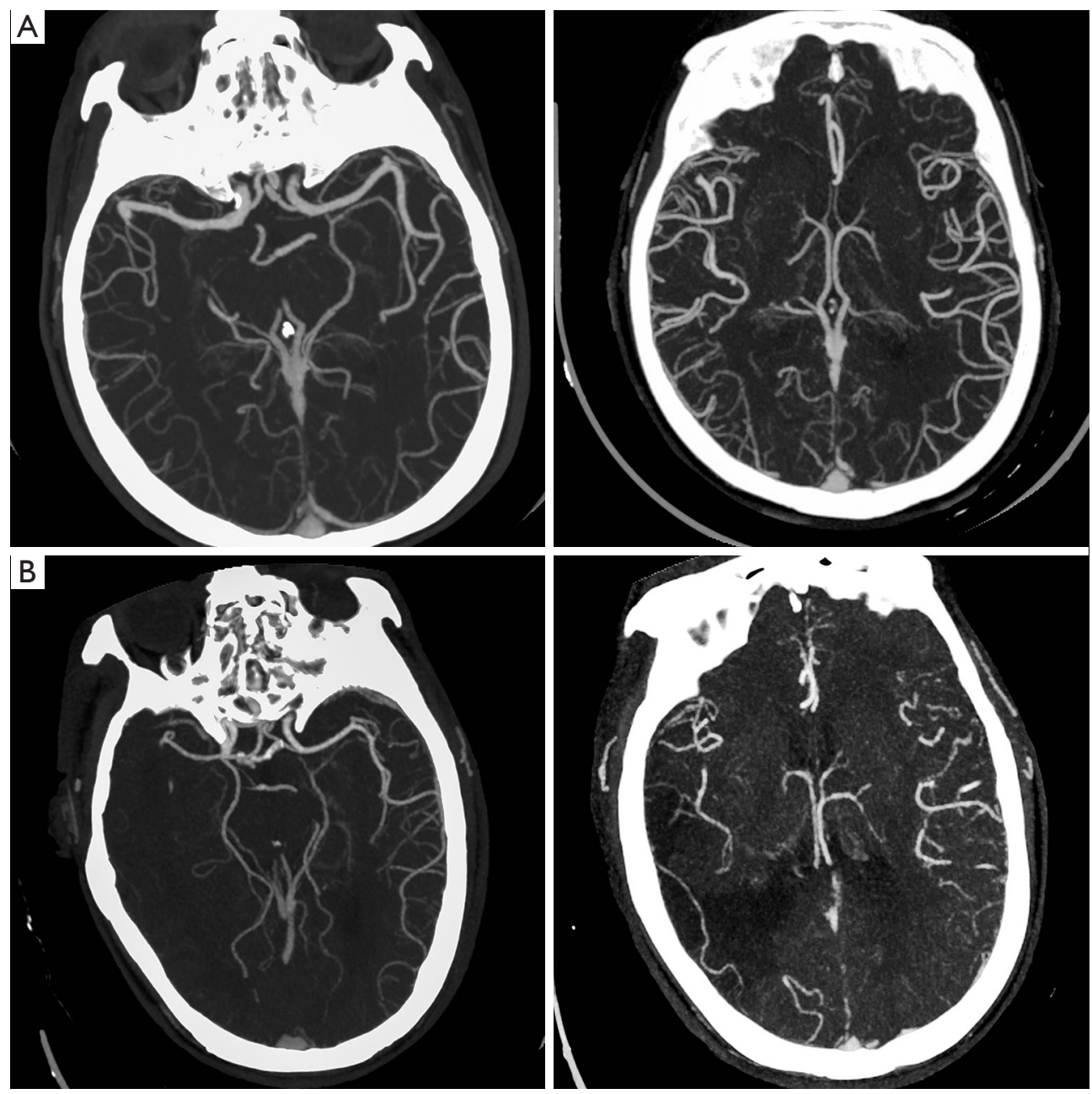

Figure 1 Patients with different LMCs status according to baseline CTA. (A) 74 years old female with good LMCs status (rLMC score $=17$ ). (B) 62 years old male with poor LMCs status (rLMC score =5). LMC, leptomeningeal collateral; CTA, computed tomographic angiography; rLMC, regional LMC.

use of anticoagulant (Table 1).

In the totality of patients, the median admission NIHSS score was 13 [8-17]. Fifty-four patients (56.3\%) went through revascularization therapy at emergency, in which 21 patients $(21.9 \%)$ were treated with intravenous thrombolysis (IVT) alone, 10 patients (10.4\%) received endovascular thrombectomy (ET) alone and 23 (24.0\%) had combined IVT and ET therapy. Besides, the median 90-day mRS was 4 [2-6]. Only 31 patients (32.3\%) achieved a good functional outcome ( $\mathrm{mRS} \leq 2), 14$ patients $(14.6 \%)$ died in hospital and 27 patients $(28.1 \%)$ died at 3 months. Notably, although there were no difference regarding the rates of receiving revascularization therapy and time-to-initiation of treatment between poor LMCs and good LMCs subgroups, patients with poor LMCs status had lower rates of favorable functional outcome $(7 / 51,13.7 \%$ versus $24 / 45,53.3 \%$, $\mathrm{P}<0.001)$ and higher rates of 3 -month mortality $(22 / 51$, $43.1 \%$ versus $5 / 45,11.1 \%, \mathrm{P}<0.001)$. Moreover, successful recanalization occurred more often in the good LMCs subgroup (12/16, 75\% versus 9/17, 52.9\%); however, the difference was not statistically significant $(\mathrm{P}=0.188)$.

\section{Associations of RGMa mRNA levels with LMCs status in patients with MCAO}

At the time of admission, there was a statistically significant difference in RGMa mRNA expression in PBMCs: 4.0 (1.5-17.6), $0.9(0.2-5.1), 2.7(0.1-4.7)$ amongst poor 
Table 1 Characteristics of the participants at baseline

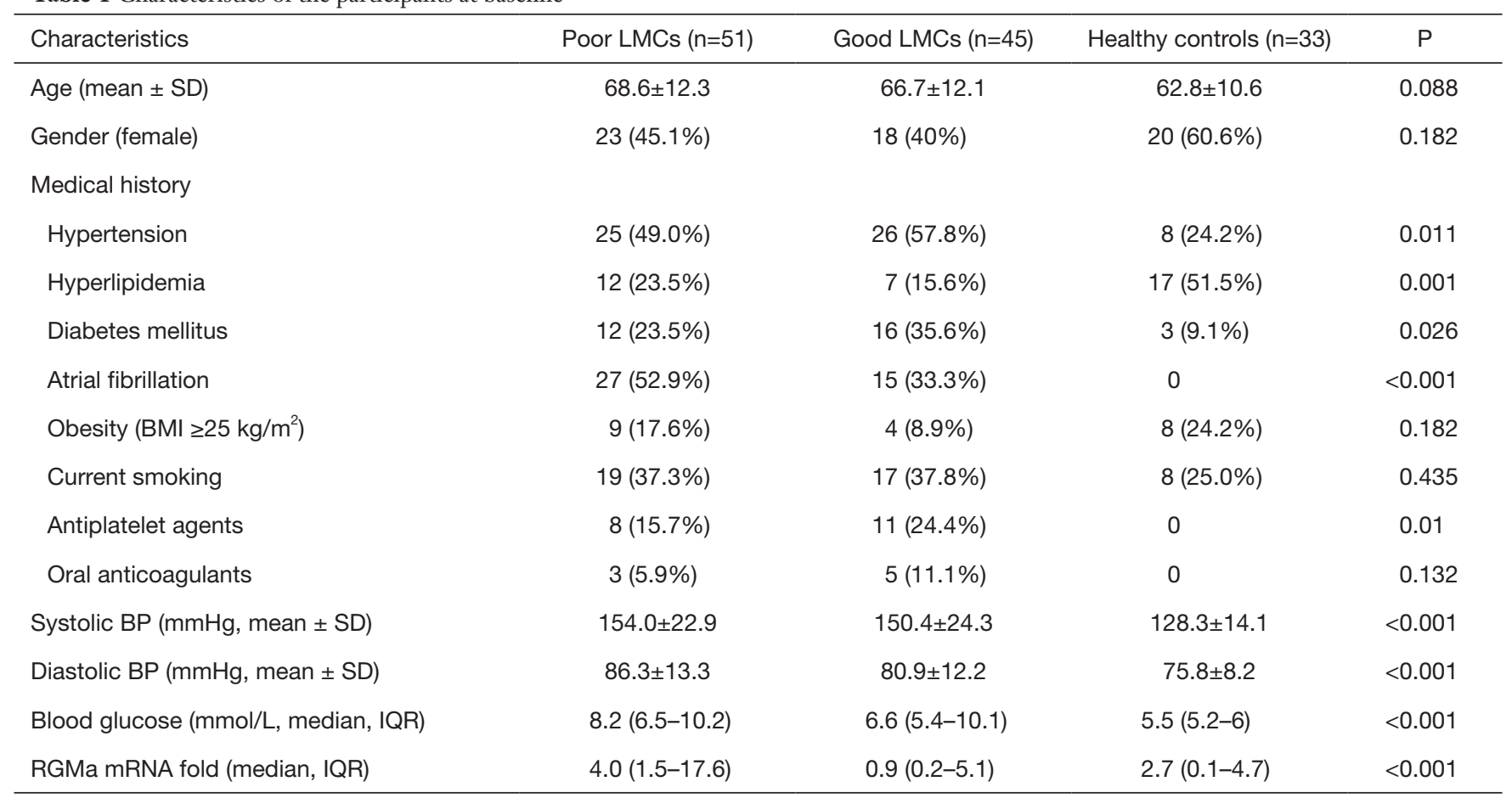

LMC, leptomeningeal collateral; RGMa, repulsive guidance molecule a.

LMCs patients, good LMCs patients and healthy controls, respectively $(\mathrm{P}<0.001)$. In the subgroup analysis, patients with poor LMCs showed significantly higher RGMa mRNA levels than patients with good LMCs status $(\mathrm{P}=0.001)$ as well as healthy controls $(\mathrm{P}=0.002)$, respectively; whereas good LMCs group showed similar baseline RGMa levels than controls $(\mathrm{P}=1.000)$ (Figure 2A).

Then, two subgroups of poor LMCs and good LMCs patients were compared to further determine the predictors for poor LMCs status. A statistically significant difference in RGMa mRNA level $(\mathrm{P}<0.001)$, NIHSS score at baseline $(\mathrm{P}=0.008)$, diastolic blood pressure on admission $(\mathrm{P}=0.04)$ and rate of LAA stroke $(\mathrm{P}=0.007)$ was observed in univariate analyses (Table 2). Multivariate logistic regression analysis revealed that RGMa mRNA level (OR 1.099, 95\% CI: $1.035-1.168, \mathrm{P}=0.002)$ as well as NIHSS score at baseline (OR 1.091, 95\% CI: 1.003-1.186, P=0.042) were independent predictors for poor LMCs status (Table 3).

We next performed the ROC analysis to test the potential usefulness of RGMa mRNA expression in PBMC for the prediction of poor LMCs status. Our data showed that RGMa levels had diagnostic prediction for impaired LMCs (AUC $=0.719,95 \%$ CI: 0.614-0.823, $\mathrm{P}<0.001$ ). Sensitivity and specificity at the cutoff value of 1.748 -fold were $72.5 \%$ and $71.1 \%$, respectively (Figure $2 B$ ).

\section{Variables associated with favorable functional outcomes in patients with MCAO}

To determine the predictors for favorable functional outcomes, patients with good and poor 90-day functional outcomes were compared. As a result, there was a statistically significant difference in the rate of poor LMCs status $(\mathrm{P}<0.001)$, baseline NIHSS score $(\mathrm{P}=0.013)$, location of stroke (right side) $(\mathrm{P}=0.034)$, location of artery occlusion (M1) $(\mathrm{P}=0.012)$, atrial fibrillation $(\mathrm{P}=0.014)$ and rate of LAA stroke $(\mathrm{P}=0.002)$ between these two groups in univariate analyses. Additionally, we found that patients with poor functional outcome tended to have higher level of RGMa mRNA (2.3, 95\% CI: $0.862-13.501)$ than those with favorable functional outcome (1.283, 95\% CI: 0.252 14.825), but the difference was not significant $(\mathrm{P}=0.216)$. In the multivariate analysis, poor LMCs status (OR 0.118, 95\% CI: $0.031-0.453, \mathrm{P}=0.002)$, NIHSS score at baseline (OR 0.893, 95\% CI: 0.798-0.999, P=0.048) and location of stroke (right side) (OR 0.19, 95\% CI: 0.053-0.683, $\mathrm{P}=0.011$ ) were independent risk factors to predict good functional outcome. 

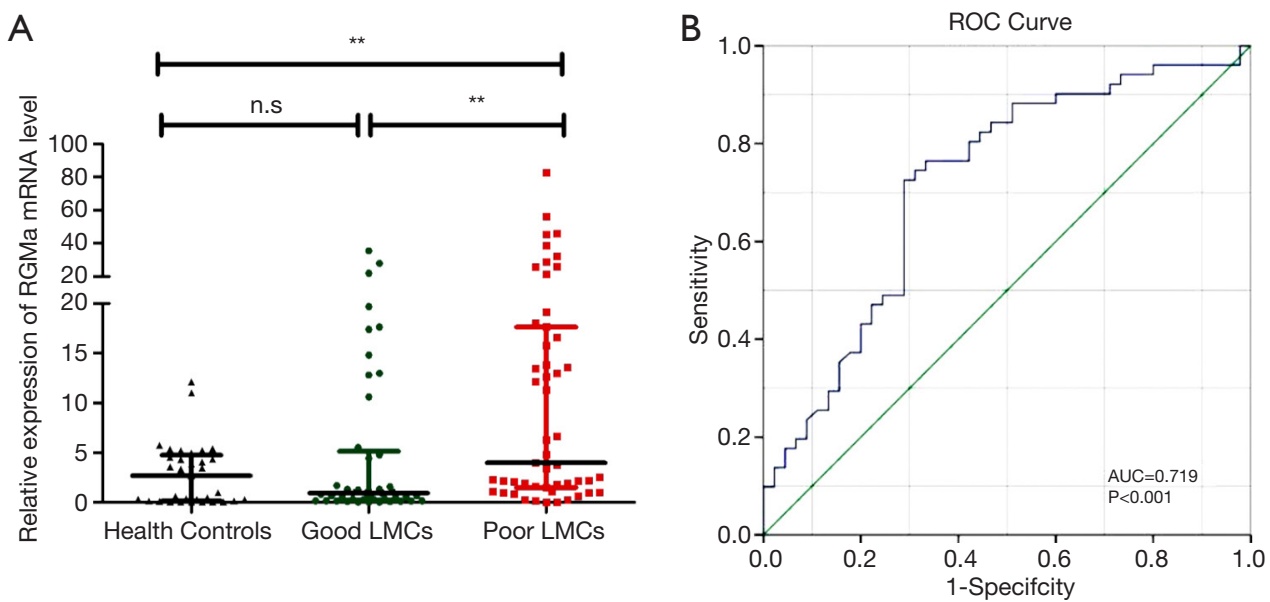

Figure 2 RGMa mRNA level in PBMCs analysis in study participants. (A) RGMa mRNA levels in AIS patients with poor LMCs, good LMCs and healthy controls. (B) ROC analysis of predicted sensitivity and 1-specifcity with mRNA levels of RGMa. The AUC for RGMa for poor LMCs status was 0.719 (95\% confidence interval, $0.614-0.823$; $\mathrm{P}<0.001$ ) with sensitivity of $72.5 \%$ and specify of $71.1 \%$ at the cutoff value of 1.748 -fold. **, $\mathrm{P}<0.01$. RGMa, repulsive guidance molecule a; PBMC, peripheral mononuclear cell; AIS, acute ischemic stroke; LMC, leptomeningeal collateral; ROC, receiver-operating characteristic.

\section{Discussion}

Penumbral 'life expectancy' in ischemic stroke is greatly dependent on the status of collateral circulation, of which the circle of Willis and leptomeningeal anastomoses are typical intracranial collateral arteries in humans $(1,2)$. In the event of MCAO, the circle of Willis is no longer able to contribute collateral blood supply and LMCs provides crucial nutritional support to the penumbra by contributing to retrograde filling of pial arteries distal to an occlusion $(3,4)$. Although there is no dispute about the importance of baseline LMCs status in setting of MCAO, questions regarding the potential causes for inter-individual variability in human LMCs are, however, unanswered. Animal studies have been informative, reporting that several genetic factors controlling arteriogenesis such as vascular endothelial growth factor a (VEGFa), chloride intracellular channel 4 (Clic4), Delta-like 4 (Dll4) and midkine impacted the extent of LMCs in ischemic disease through regulating endothelial cell function $(1,15,20-22)$. However, studies exploring the determinants of LMCs status in humans have been limited.

A key finding of the present study was that elevated RGMa mRNA in PBMCs were associated with impairment of LMCs in patients with MCAO. To the best of our knowledge, this is the first report demonstrating a connection between RGMa and LMCs status in humans, suggesting that measurement of circulatory RGMa mRNA expression in the early phase of stroke was logically expected to be useful for predicting poor LMCs status in patients with MCA occlusion, and more importantly, reflecting that RGMa might be involved in leptomeningeal arteriogenesis in AIS.

RGMa, which was originally identified as an axon repellent in the chick retinotectal system, has now emerged as a molecule which could regulate various functions in the developing and pathological CNS depending on its cellular and environmental context $(12,13,22)$. Previous studies reported that after inhibition of RGMa, angiogenesis, coupled with functional recovery was enhanced in a rat MCAO/reperfusion $(\mathrm{MCAO} / \mathrm{R})$ model, indicating that RGMa may play a negative role in angiogenesis after stroke (12). Furthermore, in vitro, the addition of recombinant RGMa significantly decreased the proliferation, migration, and tube formation of ECs, indicating that RGMa may induce endothelial dysfunction in ischemia $(13,14)$. While endothelial function also plays a main role in LMC formation $(4,7,15,16)$, a role for RGMa in leptomeningeal arteriogenesis following ischemic stroke can be hypothesized.

Furthermore, accumulating discoveries have elucidated that VEGF signaling pathway is a key element in growth and development of new arteries. Recent animal studies have reported that the level of VEGF expression strongly influenced the formation of LMCs $(1,17,20)$. Interestingly, Fujita et al. recently reported that RGMa treatment of 
Table 2 Univariate analysis of predictors of poor LMCs status

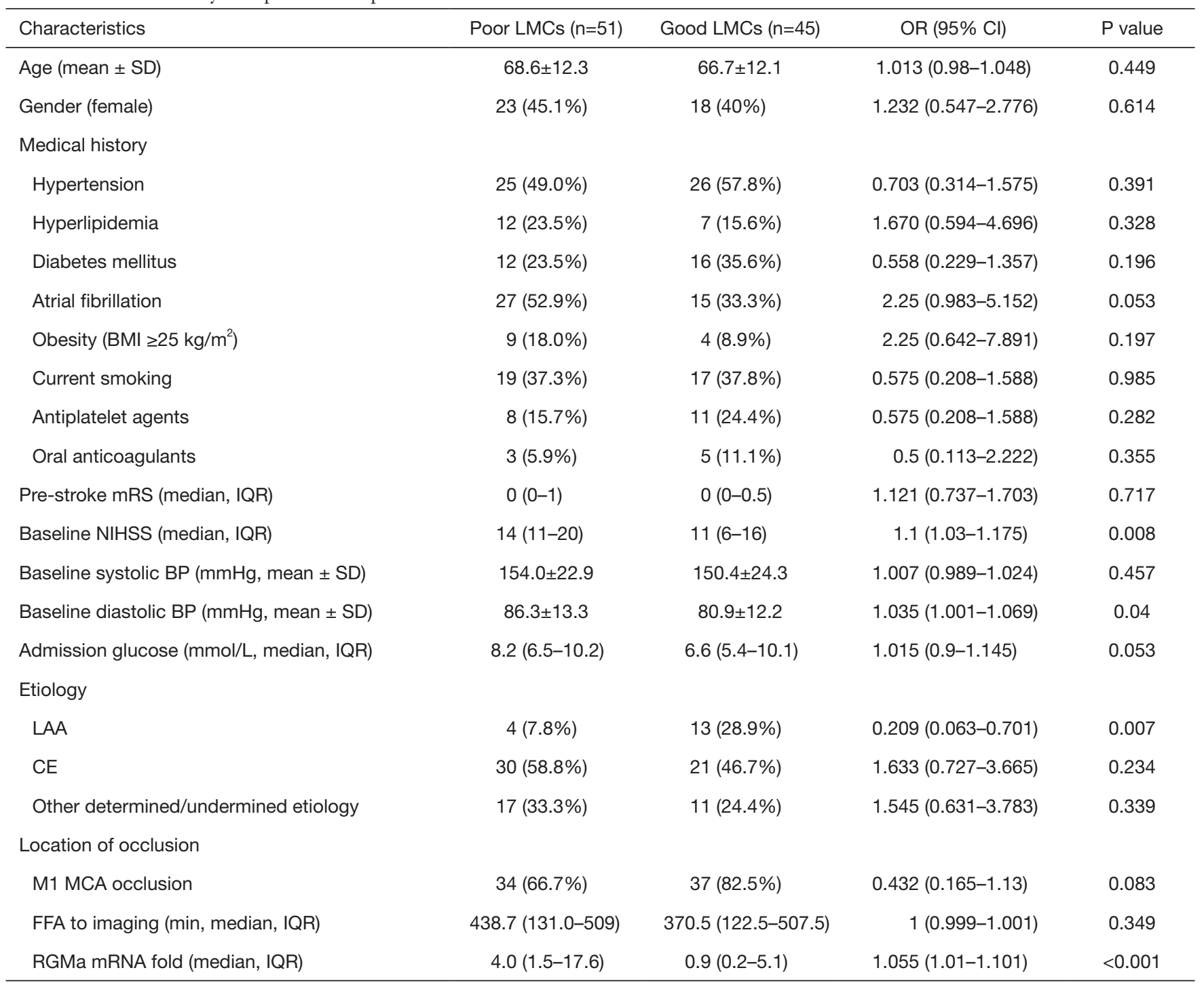

LMC, leptomeningeal collateral; RGMa, repulsive guidance molecule a.

human umbilical artery endothelial cells (HUAECs) decreased VEGF-induced phosphorylation of focal adhesion kinase (FAK) (13). Additionally, in animal models, after inhibition of RGMa, a significantly increased VEGF expression levels was detected (12). Zhang et al. implicated that RGMa was involved in VEGF-induced angiogenesis and may be an antagonist of both endogenous and exogenous VEGF (14).

Therefore, given the findings that RGMa contributes to endothelial dysfunction, together with evidence for the role of RGMa in VEGF-mediated vessel formation, we speculated that RGMa might be involved in arteriogenesis by inducing endothelial dysfunction via VEGF singling pathways, and thus determined LMCs status in ischemic stroke. Subsequent work should be performed both in vivo and in vitro to further explore the underlying role and mechanism of RGMa in leptomeningeal arteriogenesis in AIS.

Last but not the least, in spite of recent advances in revascularization therapy at emergency, MCAO still frequently leaded to adverse neurological outcomes, especially in patients with poor LMCs status. Consistent with prior studies, our study revealed that poor LMCs patients had higher baseline NIHSS score, higher mortality 
Table 3 Multivariate analysis of predictors for poor LMCs status in patients with MCAO

\begin{tabular}{lcc}
\hline Characteristics & OR $(95 \% \mathrm{Cl})$ & $\mathrm{P}$ \\
\hline Atrial fibrillation & $1.409(0.47-4.225)$ & 0.541 \\
Baseline NIHSS & $1.091(1.003-1.186)$ & 0.042 \\
Diastolic BP & $1.03(0.992-1.07)$ & 0.123 \\
Admission glucose & $0.963(0.833-1.112)$ & 0.606 \\
M1 occlusion & $0.453(0.14-1.461)$ & 0.185 \\
LAA & $0.229(0.04-1.315)$ & 0.098 \\
RGMa mRNA expression & $1.099(1.035-1.168)$ & 0.002 \\
\hline
\end{tabular}

LMC, leptomeningeal collateral; MCAO, middle cerebral artery occlusion.

rates and poorer functional outcomes. Additionally, successful recanalization was achieved more often in patients with good collateral system, even though our findings were not statistically significant. A physical trajectory for those patients with poor LMCs status has been proposed of sudden decline in functional status at stroke onset, and ending in death or survival with long-term disability. For this reason, in order to increase the quality of life, there is need for a comprehensive palliative care to provide psychosocial support, determination of patient-focused care objectives, and symptom management for these patients after stroke (23). More research and more palliative care specialists are needed in the future to better understand palliative care need of stroke patients and their families (24).

This study had some limitations. Firstly, patient enrollment from a single center, relatively small sample size, and a likely selection bias were the important limitations of our study. Additionally, given their strong clinical association of revascularization therapy with clinical outcomes in setting of MCAO, subgroups according to their treatment will be better to illustrate the variables including RGMa related with clinical outcomes, but small sample size cramped our further subgroup analysis. Finally, the measurement of RGMa mRNA expressions was based on a single sample obtained upon admission, so possible changes of RGMa mRNA levels according to the stages of AIS could not be evaluated.

\section{Conclusions}

Our study suggested that elevated PBMCs RGMa mRNA levels were associated with impaired LMCs in MCAO, indicating RGMa mRNA measurement in the early phase of stroke, together with other clinical approaches, was logically expected to be useful for predicting LMCs status in AIS patients. More importantly, a role for RGMa in leptomeningeal arteriogenesis following ischemic stroke can be hypothesized.

\section{Acknowledgments}

Funding: This work was supported by the National Natural Science Foundation of China (grant numbers 81771275).

\section{Footnote}

Reporting Checklist: The authors have completed the MDAR reporting checklist. Available at http://dx.doi.org/10.21037/ apm-20-422

Data Sharing Statement: Available at http://dx.doi. org/10.21037/apm-20-422

Peer Review File: Available at http://dx.doi.org/10.21037/ apm-20-422

Conflicts of Interest: All authors have completed the ICMJE uniform disclosure form (available at http://dx.doi. org/10.21037/apm-20-422). The authors have no conflicts of interest to declare.

Ethical Statement: The authors are accountable for all aspects of the work in ensuring that questions related to the accuracy or integrity of any part of the work are appropriately investigated and resolved. The study was conducted in accordance with the Declaration of Helsinki (as revised in 2013). The study was approved by ethics board of the First Affiliated Hospital of Chongqing Medical University (File No. 2018-066-2) and was registered in Chinese Clinical Trial Registry (File No. ChiCTR1800018569). Informed consent was taken from all the participants or their proxy.

Open Access Statement: This is an Open Access article distributed in accordance with the Creative Commons Attribution-NonCommercial-NoDerivs 4.0 International License (CC BY-NC-ND 4.0), which permits the noncommercial replication and distribution of the article with the strict proviso that no changes or edits are made and the original work is properly cited (including links to both 
the formal publication through the relevant DOI and the license). See: https://creativecommons.org/licenses/by-ncnd/4.0/.

\section{References}

1. Zhang H, Prabhakar P, Sealock R, et al. Wide genetic variation in the native pial collateral circulation is a major determinant of variation in severity of stroke. J Cereb Blood Flow Metab 2010;30:923-34.

2. Park JS, Kwak HS, Chung GH, et al. The Prognostic Value of CT-Angiographic Parameters After Reperfusion Therapy in Acute Ischemic Stroke Patients With Internal Carotid Artery Terminus Occlusion: Leptomeningeal Collateral Status and Clot Burden Score. J Stroke Cerebrovasc Dis 2018;27:2797-803.

3. Lima FO, Furie KL, Silva GS, et al. The pattern of leptomeningeal collaterals on CT angiography is a strong predictor of long-term functional outcome in stroke patients with large vessel intracranial occlusion. Stroke 2010;41:2316-22.

4. Yukami T, Yagita Y, Sugiyama Y, et al. Chronic Elevation of Tumor Necrosis Factor- $\alpha$ Mediates the Impairment of Leptomeningeal Arteriogenesis in db/db Mice. Stroke 2015;46:1657-63.

5. Brozici M, van der Zwan A, Hillen B. Anatomy and functionality of leptomeningeal anastomoses: a review. Stroke 2003;34:2750-62.

6. Menon BK, Smith EE, Coutts SB, et al. Leptomeningeal collaterals are associated with modifiable metabolic risk factors. Ann Neurol 2013;74:241-8.

7. Rizzi A, Benagiano V, Ribatti D. Angiogenesis versus arteriogenesis. Rom J Morphol Embryol 2017;58:15-9.

8. Zhang YY, Huang NN, Zhao YX, et al. Elevated Tumor Necrosis Factor-a-induced Protein 8-like 2 mRNA from Peripheral Blood Mononuclear Cells in Patients with Acute Ischemic Stroke. Int J Med Sci 2018;15:1713-22.

9. Pedard $M$, Brenière $C$, Pernet $N$, et al. Brain-derived neurotrophic factor in peripheral blood mononuclear cells and stroke outcome. Exp Biol Med (Maywood) 2018;243:1207-11.

10. Tufekci KU, Vurgun U, Yigitaslan O, et al. Follow-up Analysis of Serum TNF-Related Apoptosis-Inducing Ligand Protein and mRNA Expression in Peripheral
Blood Mononuclear Cells from Patients with Ischemic Stroke. Front Neurol 2018;9:102.

11. Zhang $\mathrm{R}, \mathrm{Wu} \mathrm{Y}$, Xie F, et al. RGMa mediates reactive astrogliosis and glial scar formation through TGF $\beta 1 /$ Smad2/3 signaling after stroke. Cell Death Differ 2018;25:1503-16.

12. Wang $Y$, Zhang R, Xing X, et al. Repulsive guidance molecule a suppresses angiogenesis after ischemia/ reperfusion injury of middle cerebral artery occlusion in rats. Neurosci Lett 2018;662:318-23.

13. Fujita Y, Yamashita T. The roles of RGMa-neogenin signaling in inflammation and angiogenesis. Inflamm Regen 2017;37:6.

14. Zhang G, Wang R, Cheng K, et al. Repulsive Guidance Molecule a Inhibits Angiogenesis by Downregulating VEGF and Phosphorylated Focal Adhesion Kinase In Vitro. Front Neurol 2017;8:504.

15. Lautz T, Lasch M, Borgolte J, et al. Midkine Controls Arteriogenesis by Regulating the Bioavailability of Vascular Endothelial Growth Factor A and the Expression of Nitric Oxide Synthase 1 and 3, EBioMedicine 2018;27:237-46.

16. Castro PR, Barbosa AS, Pereira JM, et al. Cellular and Molecular Heterogeneity Associated with Vessel Formation Processes. Biomed Res Int 2018;2018:6740408.

17. Núñez-Gómez E, Pericacho M, Ollauri-Ibáñez C, et al. The role of endoglin in post-ischemic revascularization. Angiogenesis 2017;20:1-24.

18. Adams HP, Bendixen BH, Kappelle LJ, et al. Classifcation of subtype of acute ischemic stroke. Definitions for use in a multicenter clinical trial. TOAST. Trial of Org 10172 in Acute Stroke Treatment. Stroke 1993;24:35-41.

19. Menon BK, Smith EE, Modi J, et al. Regional leptomeningeal score on CT angiography predicts clinical and imaging outcomes in patients with acute anterior circulation occlusions. AJNR Am J Neuroradiol 2011;32:1640-5.

20. Moraes F, Paye J, Mac Gabhann F, et al. Endothelial cell-dependent regulation of arteriogenesis. Circ Res 2013;113:1076-86.

21. Chalothorn D, Zhang H, Smith JE, et al. Chloride intracellular channel-4 is a determinant of native collateral formation in skeletal muscle and brain. Circ Res 2009;105:89-98.

22. Cristofaro B, Shi Y, Faria M, et al. Dll4-Notch signaling 
determines the formation of native arterial collateral networks and arterial function in mouse ischemia models. Development 2013;140:1720-9.

23. Saricam G, Akdogan D, Kahveci K. Palliative care after

Cite this article as: $\mathrm{Wu} \mathrm{Q}, \mathrm{Li} \mathrm{T}$, Gong B, Yang J, Qin X. Elevated repulsive guidance molecule-a mRNA in peripheral blood mononuclear cells are associated with impaired leptomeningeal collaterals in patients with middle cerebral artery occlusions. Ann Palliat Med 2020;9(5):2933-2942. doi: 10.21037/apm-20-422 stroke. Acta Neurol Belg. 2019;119:69-75.

24. Steigleder T, Kollmar R, Ostgathe C. Palliative Care for Stroke Patients and Their Families: Barriers for Implementation. Front Neurol. 2019;10:164. 